Open Access

\title{
Titanium versus absorbable tacks comparative study (TACS): a multicenter, non-inferiority prospective evaluation during laparoscopic repair of ventral and incisional hernia: study protocol for randomized controlled trial
}

\author{
Gianfranco Silecchia ${ }^{1,2}$, Giuseppe Cavallaro ${ }^{1,2^{*}}$, Luigi Raparelli ${ }^{3}$, Stefano Olmi ${ }^{4}$, Gianandrea Baldazzi ${ }^{5}$
} and Fabio Cesare Campanile 6

\begin{abstract}
Background: Laparoscopic repair of ventral and incisional hernias has gained popularity since many studies have reported encouraging results in terms of outcomee and recurrence. Choice of mesh and fixation methods are considered crucial issues in preventing recurrences and complications. Lightweight meshes are considered the first choice due to their biomechanical properties and the ability to integrate into the abdominal wall. Titanium helicoidal tacks still represent the "gold standard" for mesh fixation, even if they have been suggested to be involved in the genesis of post-operative pain and complications. Recently, absorbable tacks have been introduced, under the hypothesis that there will be no need to maintain a permanent fixation device after mesh integration. Nevertheless, there is no evidence that absorbable tacks may guarantee the same results as titanium tacks in terms of strength of fixation and recurrence rates. The primary end point of the present trial is to test the hypothesis that absorbable tacks are non-inferior to titanium tacks in laparoscopic incisional and ventral hernia repair (LIVHR) by lightweight polypropylene mesh, in terms of recurrence rates at 3-year follow-up. Surgical complications, post-operative stay, comfort and pain are secondary end points to be assessed.
\end{abstract}

Methods/Design: Two hundred and twenty patients with ventral hernia will be randomized into 2 groups: Group A (110) patients will be submitted to LIVHR by lightweight polypropylene mesh fixed by titanium tacks; Group B (110) patients will be submitted to LIVHR by lightweight polypropylene mesh fixed by absorbable tacks.

Discussion: A few retrospective studies have reported similar results when comparing absorbable versus non-absorbable tacks in terms of intraoperative and early post-operative outcomes. These studies have the pitfalls to be retrospective evaluation of small series of patients, and the reported results still need to be validated by larger series and prospective studies.

The aim of the present trial is to investigate and test the non-inferiority of absorbable versus non-absorbable tacks in terms of hernia recurrence rates, in order to assess whether the use of absorbable tacks may achieve the same results as non-absorbable tacks in mid-term and long-term settings.

(Continued on next page)

\footnotetext{
* Correspondence: giuseppe.cavallaro@uniroma1.it

'Department of Medico-Surgical Sciences and Biotechnologies, Sapienza University of Rome, Rome, Italy

${ }^{2}$ General Surgery Unit, ICOT Hospital, Latina, LT, Italy

Full list of author information is available at the end of the article
} 
(Continued from previous page)

Trial registration number: NCT02076984: 5 June 2014 (ClinicalTrials.gov)

Keywords: Ventral/incisional hernia repair, Laparoscopy, Lightweight polypropylene mesh, Absorbable tacks, Titanium helicoidal tacks, Hernia

\section{Background}

During the past 50 years, incisional and ventral hernia repair surgery has evolved from direct suture repair to the use of synthetic mesh to obtain a tension-free repair [1-3]. Finally, the tension-free concepts have been applied to laparoscopic surgery. Laparoscopic repair of incisional/ventral hernias (LIVHR) has gained increasing popularity, since many studies, included three metaanalyses, have reported encouraging results in terms of wound infection, hospital stay and post-operative pain, and recurrence rates comparable with the open approach [1-8].

Besides patient selection (age, sex, comorbidities, obesity, hernia site and size, eventual recurrence and type of previous abdominal surgery) and patient compliance, the choice of the mesh (bio-materials, size and shape) and fixation methods (titanium tacks, absorbable tacks, fibrin glue) are considered crucial issues to achieve optimal results and reduce complication and recurrence rates [4,5,9-12]. Lightweight meshes are often considered the first choice for hernia repair by many authors, since the evidence that the decreased density of the non-absorbable material could reduce the "foreign-body response," improve abdominal wall compliance, cause less shrinkage and enhance the integration in host tissues [13-15]. Titanium helicoidal tacks are still considered the "gold standard" for mesh fixation [16-20]. However, several complications caused by these tacks have been reported (adhesion formation and bowel perforation). Furthermore, these devices may be involved in the genesis of some post-operative pain. Recently, absorbable tacks have been introduced [21] in combination with lightweight meshes, under the assumption that permanent fixation is no longer needed after the mesh is integrated within host tissues, potentially avoiding some of the above-described tack-related complications. Nevertheless, the efficacy of the use of absorbable tacks has never been compared to titanium tacks in a prospective analysis: only a few observational studies report similar results during a mid-term post-operative follow-up [21-23].

The Italian Society of Endoscopic Surgery recently carried out a Consensus Conference with a systematic literature search on this topic and concluded that "mesh fixation with spiral tacks should be considered the standard method of fixation in laparoscopic ventral/incisional hernia repair" and that "there is not enough evidence to make any recommendation in favor or against the use of the absorbable fixing devices" [24]. Also, the International Endohernia Society published its evidence-based guidelines and came to similar conclusions. Both guideline panels found very little literature about the role of absorbable fixing devices and stressed the need for further research about it [25]. In fact, the current evidence on the use, safety and efficacy of absorbable tacks for mesh fixation is still based on few retrospective studies giving inconclusive results $[21,22]$, and a recent report from a National Register [26], which concludes that the use of absorbable tacks is related to an increased risk of hernia recurrence. The latter deals with a very large number of patients but does not provide any information either about the type of mesh or the specific kind of tacks used. The absence of this crucial information limits the usefulness of the study. The direct comparison of absorbable tacks with the current "gold standard," represented by titanium tacks appears to be the best way to assess their efficacy and safety.

Our study primary objective is to determine if absorbable tacks are non-inferior to titanium tacks for lightweight mesh fixation during LIVHR in the end point of recurrence rate, considering a follow-up of 3 years.

The secondary objectives are to evaluate the possible superiority of the absorbable tacks in improving postoperative stay, complications, pain and comfort.

Our choice of a non-inferiority trial design was based on the assumption that some of the complications related to the use of metal spiral tack could be avoided by the use of absorbable devices if their non-inferiority to the reference treatment (metal tacks) is demonstrated.

This protocol has been prepared in accordance with Standard Protocol Items: Recommendations for Interventional Trials (SPIRIT) [27] and the Consolidated Standards of Reporting Trials (CONSORT 2010) [28], taking into accounts its Extension for Nonpharmacologic Treatment Interventions [29] and the CONSORT Statement for Reporting Noninferiority and Equivalence Trials [30].

\section{Methods/Design}

\section{Trial design, locations and organizational structure}

The TACS trial is a multicentric prospective randomized study. It will involve five surgical units having extensive experience in both metallic and absorbable tacks for LIVHR (this means that each surgeon participating in 
the study usually performs more than fifty procedures per year with the devices used in the study).

The Reference Center (General Surgery Unit, ICOT Hospital, Latina, Italy) will coordinate the study and handle the data analysis and interim evaluations.

The study will develop according to the Helsinki Declaration. It obtained the Ethical Committee approval (Independent Ethical Committee "Lazio 2") on 26 May 2014 prior to registration (reference number asl_lt/15675/A001/ 2014).

\section{Study population and eligibility criteria}

All consecutive patients referred to the involved surgical units will be checked to determine eligibility, by one dedicated team member, according to the inclusion and exclusion criteria specified in an additional file (see Additional file 1).

All the eligible patients will be consecutively enrolled in the study until the sample size of 220 patients is reached. The included patients will be divided into two groups:

Group A: patients submitted to LIVHR by lightweight polypropylene mesh fixed by titanium helicoidal tacks (control group);

Group B: patients submitted to LIVHR by lightweight polypropylene mesh fixed by absorbable tacks (study group).

Each patient will be introduced to the trial by a member of the research group and receive an explanation of the study protocol (including random assignment of the fixation device that will be used during surgery). A specific informed consent regarding participation in the trial, randomization and explanation of the laparoscopic hernia repair, with no disclosure about the fixation device will be obtained and signed before enrolling in the study.

\section{Randomization, allocation concealment, and blinding}

A simple 1:1 allocation ratio randomization (without any restriction) will be guaranteed by an on-line computerized random generator. Patients will be randomly assigned to either Group A or Group B in the operating room, just before surgery.

Patients will be kept blind to the allocation status. Furthermore, post-operative controls will be performed at each center by a physician not directly involved in the study. He will be kept blind to the particular device used on each patient. Due to the nature of the intervention, the operating staff cannot be blinded to allocation but will be instructed not to disclose the allocation status of the participant at any time.
Intervention, preoperative and post-operative management All patients will be assessed preoperatively by clinical examination and abdominal computed tomography (CT) or ultrasound (US) scan to evaluate the size and number of the defect(s).

The devices used in the study (meshes and tacks) will be:

Physiomesh $^{\text {tw }}$ (Ethicon Endo-Surgery, Johnson \& Johnson, Inc.), a lightweight polypropylene mesh with double face absorbable layer engineered to be placed on the peritoneal surface

Protack $^{\text {Twx }}$ (Covidien Surgical, Mansfield, MA, USA), permanent titanium helicoidal tacks

Securestrap $^{\text {Tx }}$ (Ethicon Endo-Surgery, Johnson \& Johnson, Inc.), "U"-shaped absorbable tacks made by polydioxanone, completely resorbed by hydrolysis within 12-18 months after implantation

All the procedures will be carried out using the same surgical technique, already described in previous studies [22,23]. In particular, there will be careful adhesiolysis of the entire abdominal wall, a search for additional defects, overlapping the defect for at least $3 \mathrm{~cm}$ on each side, setting the tacks at the distance of $1.5 \mathrm{~cm}$ in a double crown configuration [31] and no use of transfixed sutures or glue. After surgery, compressive dressing will be placed for 4 weeks.

Each surgeon will be provided with a precise description of the standardized technique and a checklist of the aforementioned necessary technical items to be controlled, filled for every patient and turned back to the Reference Center. Adherence to the protocol will be assessed by review of the procedure videos: all centers will share the videos with the Reference Center. The operations performed by the Reference Center will be examined by the primary surgeon of another participating center who will check that all items on the checklist have been correctly performed.

A member of the operating team will note the patients and intervention characteristics in an online database including the following parameters:

Patient characteristics (sex, age, comorbidities, personal therapy)

Hernia features (location and size)

Mesh size

Type of tacks (absorbable/non-absorbable)

Number of tacks used

Operating time

Post-operative hospital stay

Post-operative complications during the index admission (according to the Clavien-Dindo classification) [32]. 
Acute post-operative pain (using the Visual Numeric Scale (VNS) for pain classification) [33].

Pain management (use of analgesic drugs).

There will be no difference in preoperative or postoperative care between the two groups. All patients will receive 1 dose $(1 \mathrm{~g})$ of paracetamol given intravenously at surgery and 2 more, each at 8-hour interval. Additional need for pain management will be noted on the records. The follow-up will be scheduled as follows:

1 week (clinical evaluation)

4 weeks (clinical evaluation)

6 months (clinical evaluation or phone interview)

12 months (phone call and eventual clinical evaluation)

24 months after surgery (phone call and eventual

clinical evaluation)

36 months after surgery (phone call and eventual

clinical evaluation)

During the follow-up, hernia recurrence (with eventual imaging studies) and post-operative complications will be checked. The following data will be also evaluated, using the licensed Carolinas Comfort Scale (CCS) [34,35]:

Pain (during resting, during daily activities, during walking, during exercise):

Pain control by use of analgesic drugs (which kind of drug, which dosage)

Pain at physical examination

\section{Outcome parameters}

The primary end point of the study is:

comparison of the results of the use of metallic and absorbable tacks to fix lightweight polypropylene meshes during LIVHR, in terms of hernia recurrence, to test the hypothesis of non-inferiority of absorbable tacks when compared to titanium tacks.

The endpoint will be measured by the recurrence rate at 3 years; interim evaluations will be done at 1 -year and 2-year follow-up.

The secondary end point is:

comparison of intraoperative and early post-operative outcomes, in terms of surgical complications, postoperative stay, comfort and pain

The comfort and pain parameters will be measured using the licensed CC S; surgical complications will be evaluated by the Clavien-Dindo classification [32] and on the basis of reoperation and readmission rates (1-2 and 3 years).

\section{Statistical methods}

The sample size calculation is based on the primary end point. A sample size of 110 in each group permits the achievement of a study power of $80 \%$, to detect a noninferiority margin difference between the devices used. The reference group proportion is 0.07 . The treatment group proportion is assumed to be 0.13 under the null hypothesis of inferiority. The statistic test used will be the one-sided $\mathrm{Z}$ test (unpooled). The significance level of the test will be targeted at 0.05 .

The same sample size will also be adequate to evaluate the secondary endpoints.

Statistical analysis will be performed at 1, 2 and 3 years after the first patient's enrollment by a statistician affiliated with the Reference Center.

\section{Dissemination policy}

The results of the TACS trial will be presented at international medical meetings concerning the corresponding fields of interest. Publications are planned in surgical scientific journals. The results will be disseminated regardless of the magnitude or direction of the measured effects.

\section{Good clinical practice and ethical approval}

The study will develop according to the Helsinki Declaration. It obtained the Ethical Committee approval (Independent Ethical Committee "Lazio 2") on 26 May 2014 prior to registration (reference number asl_lt/15675/ A001/2014). Any modifications to the protocol that may impact on the conduct of the study, potential benefit to the patient or may affect patient safety will require a formal amendment to the protocol and a new approval of the Ethical Committee according to local regulations.

\section{Discussion}

In recent years, lightweight meshes have been proposed for the treatment of abdominal wall hernias, both in open and laparoscopic repair, to reduce the mesh-related and/or mesh fixation-related complications [13]. Decreasing the density of non-absorbable material, a reduced "foreign-body response" has been demonstrated. This results in an improved abdominal wall compliance and less mess shrinkage, thus allowing a better integration of the mesh in the host tissues $[14,15]$.

This "lightweight approach" is also based on the results of studies concerning the abdominal wall biomechanics [36-39].

Among the large number of meshes and materials utilized for LIVHR, macropore lightweight polypropylene has been demonstrated to be more efficient than other materials in terms of inflammatory response and mesh integration in host tissues [38-42].

A crucial issue in LIVHR is the choice of the mesh fixation method. Titanium helicoidal tacks are most frequently 
used, in a double crown configuration [43-46]; they guarantee excellent fixation strength [46] but remain in the body permanently, and have been associated with severe complications. Dense adhesion formation, erosion of tacks in hollow viscera and the formation of so-called "tack hernias" have been reported $[16,17,47]$. However, the most clinically relevant negative aspect appears to be the increased acute and chronic post-operative pain, as reported by several studies [22,23,40-42]. Furthermore, permanent fixation devices seem not to be necessary while using lightweight macropore meshes, as they become integrated into the abdominal wall and may not need to be permanently secured [22,23]. Nevertheless, titanium tacks remain the most common fixation device for LIVHR $[47,48]$. Recently, absorbable tacks have been introduced [21] to improve biocompatibility, reduce the risk of endoperitoneal adhesions due to the presence of metallic tacks, and reduce mid-term and long-term post-operative pain. The rationale for the use of absorbable tacks in LIVHR for lightweight macropore mesh fixation is mainly the biomechanical mesh features since the integration of these prostheses into the abdominal wall may not need permanent fixation devices. To date, only 2 studies dealing with the clinical use of absorbable tacks with lightweight mesh have been published [21,22]. They report similar intraoperative and early post-operative outcomes when comparing absorbable and non-absorbable tacks [22]. However, these are observational studies on small series of patients, and the reported results still need to be validated.

The aim of the present trial is to compare the results of the use of metallic and absorbable tacks to fix lightweight polypropylene meshes during LIVHR, in terms of hernia recurrence, in order to test the hypothesis the non-inferiority of absorbable tacks when compared to titanium tacks.

Furthermore, the authors will compare acute and chronic post-operative pain after the use of titanium and absorbable tacks.

If the non-inferiority of the absorbable fixation devices was assessed, their use could be encouraged and their possible advantages in terms of reduction of complications and post-operative pain could be further investigated.

\section{Trial registration and protocol version}

Primary registry and trial identifying number: Clinical Trials.gov NCT02076984.

Date of registration in the primary registry: 5 June 2014. Protocol version: version 1.1 issued on 20 January 2015.

\section{Trial status}

Patient enrollment started on 1 June 2014 after tEthical Committee approval.

\section{Additional file}

Additional file 1: Table showing inclusion and exclusion criteria for eligibility in the study.

\section{Abbreviations}

CCS: Carolinas Comfort Scale; CT: computed tomography; LIVHR: laparoscopic incisional/ventral hernia repair; US: ultrasound; VNS: Visual Numeric Scale.

\section{Competing interests}

The authors declare that they have no competing interests.

\section{Authors' contributions}

FCC and GC: conceived the design of the present study and wrote the manuscript. GC is also the scientific coordinator of the participating centers; FCC is also the local coordinator of one of the surgical units involved. All authors read and approved the final manuscript. GS: is the principal investigator, coordinator of the Reference Center, contributed to the study design and critically revised the manuscript. LR, SO, GB are local coordinators of involved center. All authors contributed to the refinement of the study protocol and approved the final manuscript.

\section{Acknowledgements}

The study received neither industry funding nor financial/non-financial support by any organization that may have an interest in its results. However, the authors disclose that they have individually received occasional travel grants for the participation in national and international congresses from Johnson \& Johnson and Covidien in the past 3 years. The mentioned funding is not related in any way to the present study

\section{Author details}

${ }^{1}$ Department of Medico-Surgical Sciences and Biotechnologies, Sapienza University of Rome, Rome, Italy. ${ }^{2}$ General Surgery Unit, ICOT Hospital, Latina, LT, Italy. ${ }^{3}$ General Surgery Unit, GB Grassi Hospital, Ostia, RM, Italy. ${ }^{4}$ General Surgery Unit, Gruppo Ospedaliero San Donato, Milan, Italy. ${ }^{5}$ General and Mini-Invasive Surgery Unit, Abano Terme Hospital, Abano Terme, PD, Italy.

${ }^{6}$ General Surgery Unit, Andosilla Hospital, Civita Castellana, VT, Italy.

Received: 19 February 2015 Accepted: 22 May 2015

Published online: 04 June 2015

\section{References}

1. Melvin WS, Renton D. Laparoscopic ventral hernia repair. World J Surg. 2011;35:1496-9.

2. Olmi S, Scaini A, Cesana GC, Erba L, Croce E. Laparoscopic versus open incisional hernia repair: an open randomized controlled study. Surg Endosc. 2007;21:555-9.

3. Sauerland S, Walgenbach M, Habermalz B, Seiler CM, Miserez M. Laparoscopic versus open surgical techniques for ventral or incisional hernia repair. Cochrane Database Syst Rev. 2011;3, CD007781.

4. Forbes SS, Eskicioglu C, McLeod RS, Okrainec A. Meta-analysis of randomized controlled trials comparing open and laparoscopic ventral and incisional hernia repair with mesh. Br J Surg. 2009;96:851-8.

5. Sajid MS, Bokhari SA, Mallick AS, Cheek E, Baig MK. Laparoscopic versus open repair of incisional/ventral hernia: a meta-analysis. Am J Surg. 2009;197:64-72.

6. Pierce RA, Spitler JA, Frisella MM, Matthews BD, Brunt LM. Pooled data analysis of laparoscopic vs. open ventral hernia repair: 14 years of patient data accrual. Surg Endosc. 2007;21:378-86.

7. Nardi MJ, Millo P, Brachet-Contul R, Fabozzi M, Persico F, Roveroni M, et al. Laparoscopic incisional and ventral hernia repair (LIVHR) with Parietex Composite mesh. MITAT. 2012;21:173-80.

8. Zhang Y, Zhou H, Chai Y, Cao C, Jin K, Hu Z. Laparoscopic versus open incisional and ventral hernia repair: a systematic review and meta-analysis. World J Surg. 2014;38:2233-40.

9. Reynvoet E, Berrevoet F, De Somer F, Vercauteren G, Vanoverbeke I, Chiers $\mathrm{K}$, Troisi R. Tensile strength testing for resorbable mesh fixation systems in laparoscopic ventral hernia repair. Surg Endosc. 5 April 2012. in press, doi 10.1007/s00464-012-224-5. 
10. Chevrel JP, Rath AM. Classification of incisional hernias of the abdominal wall. Hernia. 2000:4:7-11.

11. Deeken CR, Faucher KM, Matthews BD. A review of the composition, characteristics, and effectiveness of barrier mesh prostheses utilized for laparoscopic ventral hernia repair. Surg Endosc. 2012;26:566-75.

12. Cobb WS, Kercher KW, Heniford BT. The argument for lightweight polypropylene mesh in hernia repair. Surg Innov. 2005;12:T1-7.

13. Shankaran $\mathrm{V}$, Weber DJ, Reed 2 nd RL, Luchette FA. A review of available prosthetics for ventral hernia repair. Ann Surg. 2011;253:16-26.

14. Cobb WS, Burns JM, Kercher KW, Matthews BD, James Norton H, Todd HB. Normal intraabdominal pressure in healthy adults. J Surg Res. 2005;129:231-5.

15. Klinge $U$, Junge $K$, Stumpf M, AP AP, Klosterhalfen B. Functional and morphological evaluation of a low-weight, monofilament polypropylene mesh for hernia repair. J Biomed Mater Res. 2002;63:129-36.

16. Eriksen JR, Poornoroozy P, Jørgensen LN, Jacobsen B, Friis-Andersen HU, Rosenberg J. Pain, quality of life and recovery after laparoscopic ventral hernia repair. Hernia. 2008;12:483-91.

17. Eriksen JR, Bech Jl, Linnemann D, Rosenberg J. Laparoscopic intraperitoneal mesh fixation with fibrin sealant (Tisseel) vs. titanium tacks: a randomised controlled experimental study in pigs. Hernia. 2008;12:483-91.

18. Rosen MJ, Duperier T, Marks J, Onders R, Hardacre J, Ponsky J, et al. Prospective randomized double-blind placebo-controlled trial of postoperative elastomeric pain pump devices used after laparoscopic ventral hernia repair. Surg Endosc. 2009;23:2637-43.

19. Lerdsirisopon S, Frisella MM, Matthews BD, Deeken CR. Biomechanical evaluation of potential damage to hernia repair materials due to fixation with helical titanium tacks. Surg Endosc. 2011;25:3890-7.

20. Ferrari G, Bertoglio C, Magistro C, Girardi V, Mazzola M, Di Lernia S, et al. Laparoscopic repair for recurrent incisional hernias: a single institute experience of 10 years. Hernia. 2013;17:573-80.

21. Lepere M, Benchetrit S, Bertrand JC, Chalbet JY, Combier JP, Detruit B, et al Laparoscopic resorbable mesh fixation. Assessment of an innovative disposable instrument delivering resorbable fixation device: $I-$ Clip $^{T M}$. Final results of a prospective multicentre clinical trial. Hernia. 2008;12:177-83.

22. Cavallaro G, Campanile FC, Rizzello M, Greco F, lorio O, lossa A, et al. Lightweight polypropylene mesh fixation in laparoscopic incisional hernia repair. MITAT. 2013;22:283-7.

23. Cavallaro G, Campanile F, Rizzello M, Greco F, lorio O, Angelis FD, et al. Laparoscopic incisional hernia repair by lightweight polypropylene mesh with resorbable coating: technical notes, preliminary results. Chirurgia. 2013;108:304-11

24. Cuccurullo D, Piccoli M, Agresta F, Magnone S, Corcione F, Stancanelli V, et al. Laparoscopic ventral incisional hernia repair: evidence-based guidelines of the first Italian consensus conference. Hernia. 2013;17:557-66.

25. Bittner R, Bingener-Casey J, Dietz U, Fabian M, Ferzli GS, Fortelny RH, et al. International Endohernia Society (IEHS). Guidelines for laparoscopic treatment of ventral and incisional abdominal wall hernias (International Endohernia Society (IEHS)-part 1. Surg Endosc. 2014;28:2-29.

26. Christoffersen MW, Brandt E, Helgstrand F, Westen M, Rosenberg J, Kehlet $H_{\text {, }}$ et al. Recurrence rate after absorbable tack fixation of mesh in laparoscopic incisional hernia repair. Br J Surg. 2015;102:541-7.

27. Chan AW, Tetzlaff JM, Altman DG, Laupacis A, Gøtzsche PC, Krleža-Jerić K, et al. SPIRIT 2013 statement: defining standard protocol items for clinical trials. Ann Intern Med. 2013;158:200-7.

28. Schulz KF, Altman DG, Moher D. CONSORT Group. CONSORT 2010 statement: updated guidelines for reporting parallel group randomized trials. Ann Intern Med. 2010;152:726-32.

29. Boutron I, Moher D, Altman DG, Schulz KF, Ravaud P. for the CONSORT Group. Methods and processes of the CONSORT Group: example of an extension for trials assessing nonpharmacologic treatments. Ann Intern Med. 2008;148:295-309.

30. Piaggio G, Elbourne DR, Pocock SJ, Evans SJ, Altman DG, CONSORT Group. Reporting of noninferiority and equivalence randomized trials: extension of the CONSORT 2010 statement. JAMA. 2012;308:2594-604.

31. Muysoms F, Vander Mijnsbrugge G, Pletinckx P, Boldo E, Jacobs I, Michiels $M$, et al. Randomized clinical trial of mesh fixation with "double crown" versus "sutures and tackers" in laparoscopic ventral hernia repair. Hernia. 2013;17:603-12.

32. Dindo D, Demartines N, Clavien PA. Classification of surgical complications: a new proposal with evaluation in a cohort of 6336 patients and results of a survey. Ann Surg. 2004;240:205-13.
33. Ritter PL, González VM, Laurent DD, Lorig KR. Measurement of pain using the visual numeric scale. J Reumathol. 2006:33:574-80.

34. Nielsen K, Poelman MM, den Bakker FM, van der Ploeg T, Bonjer HJ, Schreurs WH. Comparison of the Dutch and English versions of the Carolinas Comfort Scale: a specific quality-of-life questionnaire for abdominal hernia repairs with mesh. Hernia. 2014;18:459-64.

35. Heniford BT, Walters AL, Lincourt AE, Novitsky YW, Hope WW, Kercher KW. Comparison of generic vs specific quality-of-life scales for mesh hernia repairs. J Am Coll Surg. 2008;206:638-44.

36. Welty G, Klinge U, Klosterhalfen B, Kasperk R, Schumpelick V. Functional impairment and complaints following incisional hernia repair with different polypropylene meshes. Hernia. 2001;5:142.

37. Szymczak C, Lubowiecka I, Tomaszewska A, Smietański M. Investigation of abdomen surface deformation due to life excitation: implications for implant selection and orientation in laparoscopic ventral hernia repair. Clin Biomech (Bristol, Avon). 2012;27(2):105-10.

38. Smietański M, Bury K, Tomaszewska A, Lubowiecka I, Szymczak C. Biomechanics of the front abdominal wall as a potential factor leading to recurrence with laparoscopic ventral hernia repair. Surg Endosc. 2012;26(5):1461-7.

39. Junge $K$, Klinge $U$, Prescher A, Giboni P, Niewiera M, Schumpelick V. Elasticity of the anterior abdominal wall and impact for reparation of incisional hernias using mesh implants. Hernia. 2001;5:113-8.

40. Rosch R, Junge K, Schachtrupp A, Klinge U, Klosterhalfen B, Schumpelick V. Mesh implants in hernia repair. Inflammatory cell response in a rat model. Eur Surg Res. 2003:35:161-6.

41. Eriksen JR. Pain and convalescence following laparoscopic ventral hernia repair. Dan Med Bull. 2011;58:B4369.

42. Eriksen JR, Poornoroozy P, Jørgensen LN, Jacobsen B, Friis-Andersen HU, Rosenberg J. Pain, quality of life and recovery after laparoscopic ventral hernia repair. Hernia. 2009;13:13-21.

43. Wassenaar E, Schoenmaeckers E, Raymakers J, van der Palen J, Rakic S. Mesh-fixation method and pain and quality of life after laparoscopic ventral or incisional hernia repair: a randomized trial of three fixation techniques. Surg Endosc. 2010;24:1296-302.

44. Carbajo A, del Olmo JC M, Blanco JL, Toledano M, De la Cuesta C, Ferreras C, et al. Laparoscopic approach to incisional hernia. Lessons learned from 270 patients over 8 years. Surg Endosc. 2003;17:118-22.

45. Heniford T, Park A, Ramshaw BJ, Voeller G. Laparoscopic repair of ventral hernias; nine years' experience with 850 consecutive hernias. Ann Surg 2003;238:391-400.

46. LeBlanc KA. Laparoscopic incisional hernia repair: are transfascial sutures necessary? A review of the literature. Surg Endosc. 2007;21:508-13.

47. Reynvoet E, Berrevoet F. Pros and cons of tacking in laparoscopic hernia repair. Surg Technol Int. 2014;25:136-40.

48. Hollinsky C, Kolbe T, Walter I, Joachim A, Sandberg S, Koch T, et al. Tensile strength and adhesion formation of mesh fixation systems used in laparoscopic incisional hernia repair. Surg Endosc. 2010;24:13181324.

\section{Submit your next manuscript to BioMed Central and take full advantage of:}

- Convenient online submission

- Thorough peer review

- No space constraints or color figure charges

- Immediate publication on acceptance

- Inclusion in PubMed, CAS, Scopus and Google Scholar

- Research which is freely available for redistribution

Submit your manuscript at www.biomedcentral.com/submit

C Biomed Central 